\title{
Holographic entanglement negativity for conformal field theories with a conserved charge
}

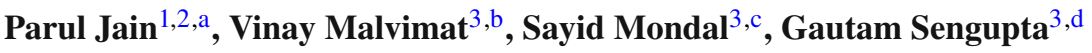 \\ ${ }^{1}$ Dipartimento di Fisica, Università di Cagliari Cittadella Universitaria, 09042 Monserrato, Italy \\ ${ }^{2}$ INFN, Sezione di Cagliari, Cagliari, Italy \\ ${ }^{3}$ Department of Physics, Indian Institute of Technology Kanpur, Kalyanpur 208016, India
}

Received: 5 August 2018 / Accepted: 29 October 2018 / Published online: 9 November 2018

(C) The Author(s) 2018

\begin{abstract}
We study the application of our recent holographic entanglement negativity conjecture for mixed states of adjacent subsystems in conformal field theories with a conserved charge. In this context we obtain the holographic entanglement negativity for zero and finite temperature mixed state configurations in $d$-dimensional conformal field theories dual to bulk extremal and non extremal charged $A d S_{d+1}$ black holes. Our results conform to quantum information theory expectations and constitute significant consistency checks for our conjecture.
\end{abstract}

\section{Introduction}

In recent times quantum entanglement has emerged as an important facet of modern fundamental physics, relating diverse fields ranging from many body theory to issues of quantum gravity and black holes. In this context the measure of entanglement entropy has played a crucial role in the characterization of quantum entanglement for bipartite pure states. In quantum information theory the entanglement entropy is defined as the von Neumann entropy of the reduced density matrix for the corresponding subsystem. Significantly, the entanglement entropy may be computed through a replica technique for bipartite states in $(1+1)$ dimensional conformal field theories $\left(C F T_{1+1}\right)$ as described in [1,2]. Interestingly Ryu and Takayanagi in a seminal work [3-5] proposed an elegant holographic entanglement entropy conjecture for bipartite states of dual $d$-dimensional conformal field theories $\left(C F T_{d}\right)$ in the framework of the $A d S / C F T$ correspondence. The Ryu-Takayanagi conjec-

\footnotetext{
a e-mail: parul.jain@ca.infn.it

b e-mail: vinaymm@iitk.ac.in

c e-mail: sayidphy@iitk.ac.in

de-mail: sengupta@iitk.ac.in
}

ture inspired extensive investigations in various aspects of entanglement in holographic $C F T$ s [5-11] (and references therein). A proof of this conjecture from a bulk perspective was subsequently developed in a series of communications, first for the $A d S_{3} / C F T_{2}$ scenario and later extended to a generic $A d S_{d+1} / C F T_{d}$ framework [12-17].

It is well known however in quantum information theory that entanglement entropy fails to characterize mixed state entanglement as it receives contributions which are irrelevant to the entanglement of the configuration in question. Hence characterization of mixed state entanglement was a complex and subtle issue which required the introduction of suitable measures. In a seminal work Vidal and Werner [18] addressed this critical issue and proposed a computable measure for characterizing the upper bound on the distillable entanglement for bipartite mixed states, termed as entanglement negativity. It could be shown that this measure is non convex and an entanglement monotone [19]. Interestingly, in a series of communication the authors in [20-22] computed the entanglement negativity for several bipartite mixed state configurations in $C F T_{1+1}$ s employing a suitable replica technique.

The above discussion naturally leads to the issue of a holographic characterization for the entanglement negativity of bipartite pure and mixed states in dual $C F T \mathrm{~s}$, in terms of the bulk geometry through the $\operatorname{AdS} / C F T$ correspondence. There were several attempts in the literature $[23,24]$ to address this issue and despite significant progress a clear elucidation of a holographic characterization for the entanglement negativity remained a crucial open problem. In the recent past, two of the present authors (VM and GS) in the collaboration [25-27] (CMS), proposed a holographic entanglement negativity conjecture for bipartite states in the dual $C F T$ s. According to their conjecture, the holographic negativity characterizing the entanglement of a simply connected single subsystem with the rest of the system, is described by 
a specific algebraic sum of the areas of co dimension two bulk static minimal surfaces (lengths of space like geodesics in the $A d S_{3} / C F T_{2}$ scenario) anchored on appropriate subsystems. In the $A d S_{3} / C F T_{2}$ context [25] their conjecture could exactly reproduce the universal part of the corresponding $C F T_{1+1}$ replica technique results, in the large central charge limit. Furthermore their analysis was strongly confirmed through a large central charge analysis of the entanglement negativity in $C F T_{1+1}$ employing the monodromy technique in [28]. The corresponding higher dimensional extension of the conjecture was substantiated through strong consistency checks involving applications to specific examples [26]. Interestingly, this reproduced certain universal features of the holographic entanglement negativity for the corresponding $A d S_{3} / C F T_{2}$ scenario [25]. However we should mention here that a formal bulk proof for their conjecture along the lines of [17] remains a non trivial open issue which needs to be addressed.

Recently, in a subsequent communication [29] the present authors proposed an independent holographic entanglement negativity conjecture for a bipartite mixed state configuration of adjacent intervals in a dual $C F T_{1+1}$. The conjecture involved a specific algebraic sum of the lengths of space like geodesics in the dual bulk $A d S_{3}$ configuration which are anchored on appropriate intervals. Interestingly, this reduced to the holographic mutual information between the two intervals upto a numerical constant. ${ }^{1}$ Remarkably, as earlier [2527], in this case also the holographic entanglement negativity exactly reproduced the universal part of the corresponding $C F T_{1+1}$ results in the large central charge limit.

A higher dimensional extension of the above conjecture for the mixed state of adjacent subsystems in a holographic $C F T_{d}$ was proposed subsequently in [32]. As earlier this involved a specific algebraic sum of the areas of codimension two bulk static minimal surfaces anchored on the respective subsystems in the dual $C F T_{d}$. This extension was substantiated through applications to specific higher dimensional examples constituting strong consistency checks for the holographic conjecture. These involved the computation of the holographic entanglement negativity for mixed states of adjacent subsystems described by rectangular strip geometries in $C F T_{d}$ s dual to bulk pure $A d S_{d+1}$ geometry and $A d S_{d+1}$-Schwarzschild black hole. Quite interestingly, for the finite temperature case involving the dual $A d S_{d+1^{-}}$Schwarzschild black hole, the holographic entanglement negativity scales as the area of the entangling surface in a high temperature approximation. Note that this is unlike the case of entanglement entropy which scales as the volume of

\footnotetext{
${ }^{1}$ Note that this matching between the universal. part of the entanglement negativity and the mutual information for the case of adjacent intervals has also been reported for time dependent situations following both local and global quenches in a $C F T_{1+1}[30,31]$.
}

the subsystem at high temperatures [33]. For the holographic entanglement negativity on the other hand, all volume dependent thermal terms cancel out leading to a purely area dependent expression. This conforms to the standard quantum information theory expectations for the entanglement negativity measure. Interestingly, the area law for entanglement negativity has also been reported for condensed matter system such as the finite temperature quantum spin model and the two dimensional harmonic lattice $[34,35]$. Subsequently, a covariant version of the holographic entanglement negativity conjecture described in [29], was proposed in [36] for time dependent mixed state configurations of adjacent intervals in a dual $C F T_{1+1}$.

In this article we further substantiate the higher dimensional $A d S_{d+1} / C F T_{d}$ extension of the holographic entanglement negativity conjecture described above, with additional non trivial consistency checks involving the application to distinct examples. This involves zero and finite temperature mixed state configurations of adjacent subsystems with rectangular strip geometries in holographic $C F T_{d} \mathrm{~s}$ with a conserved charge, dual to bulk extremal and non extremal $\mathrm{RN}-A d S_{d+1}$ black holes. Unlike for the case of $C F T_{d}$ s dual to $A d S_{d+1}$-Schwarzschild black holes [32], the holographic entanglement negativity for the present case necessitates perturbative expansions involving non trivial limits of the relevant parameters (see, also [8,37] for the corresponding case of entanglement entropy). In order to illustrate this we initially consider the $A d S_{4} / C F T_{3}$ examples for simplicity and subsequently describe the more general $A d S_{d+1} / C F T_{d}$ scenario.

In this context we first compute the holographic entanglement negativity for bipartite mixed states of adjacent subsystems in $\mathrm{CFT}_{3}$ s dual to bulk non-extremal and extremal $\mathrm{RN}-\mathrm{Ad} \mathrm{S}_{4}$ black holes. We demonstrate that the holographic entanglement negativity following from our conjecture in the various limits of the relevant parameters conform to quantum information expectations. Hence these serve as significant consistency checks for the universality of our conjecture although a bulk proof remains an outstanding open issue. The corresponding $A d S_{d+1} / C F T_{d}$ case necessitates the perturbative description of the holographic entanglement negativity involving various limits of a distinct set of parameters. However the results of this exercise are similar to the previous case of $A d S_{4} / C F T_{3}$ and lead to identical conclusions in the appropriate limits for the relevant parameters.

The article is organized as follows. In Sect. 2 we describe our holographic entanglement negativity conjecture for mixed state configurations of adjacent subsystems characterized by a rectangular strip geometry in $C F T_{d}$ s dual to bulk $A d S_{d+1}$ configurations. Subsequently, in Sect. 3 we compute the holographic entanglement negativity for mixed states of adjacent subsystems in the $A d S_{4} / C F T_{3}$ scenario. In Sect. 4 we obtain the holographic entanglement negativity 
for the required mixed states in the $A d S_{d+1} / C F T_{d}$ scenario. In the final Sect. 5 we present a summary of our results and conclusions.

\section{Holographic entanglement negativity conjecture}

In this section we briefly review the holographic entanglement negativity conjecture for a bipartite mixed state configuration of adjacent subsystems in dual $C F T$ s. To this end we first describe the holographic entanglement negativity conjecture for the mixed state configuration above in the context of the $A d S_{3} / C F T_{2}$ scenario [29]. Following this we briefly discuss the extension of our conjecture to a generic higher dimensional $A d S_{d+1} / C F T_{d}$ scenario [32].

The entanglement negativity for a bipartite mixed state configuration in a $C F T_{1+1}$ may be obtained through a suitable replica technique as described in [20-22]. This involves the spatial tripartition of the $C F T_{1+1}$ into the intervals $A_{1}$ and $A_{2}$ such that $A=A_{1} \cup A_{2}$, and the rest of the system is $A^{c}=\left(A_{1} \cup A_{2}\right)^{c}$. The entanglement negativity is then defined as

$\mathcal{E}=\lim _{n_{e} \rightarrow 1} \ln \operatorname{Tr}\left(\rho_{A}^{T_{2}}\right)^{n_{e}}$,

where $\rho_{A}^{T_{2}}$ is the partial transpose with respect to the interval $A_{2}$ for the reduced density matrix $\rho_{A}$ and the replica limit described as $n_{e} \rightarrow 1$ is an analytic continuation for even sequences of $n_{e}$ to $n_{e}=1$.

For the specific mixed state configuration of adjacent intervals $A_{1}$ and $A_{2}$ it could be shown that the quantity $\operatorname{Tr}\left(\rho_{A}^{T_{2}}\right)^{n_{e}}$ in Eq. (1) may be expressed as a three point twist correlator on the complex plane which is fixed by the conformal symmetry as

$$
\begin{aligned}
& \operatorname{Tr}\left(\rho_{A}^{T_{2}}\right)^{n_{e}}=\left\langle\mathcal{T}_{n_{e}}\left(z_{1}\right) \overline{\mathcal{T}}_{n_{e}}^{2}\left(z_{2}\right) \mathcal{T}_{n_{e}}\left(z_{3}\right)\right\rangle
\end{aligned}
$$

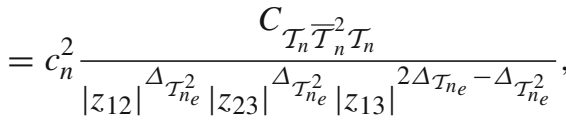

where $\left|z_{i j}\right|=\left|z_{i}-z_{j}\right|$, and $\Delta_{\tau_{n_{e}}^{2}}$ and $\Delta_{\tau_{n_{e}}}$ are the scaling dimensions of the twist fields $\tau_{n_{e}}^{2}$ and $\tau_{n_{e}}$ respectively. In the large central charge limit this three point twist correlator Eq. (2) may be expressed in terms of the lengths of bulk space like geodesics anchored on the appropriate intervals, through the standard AdS/CFT dictionary as follows [29]

$$
\begin{aligned}
& \left\langle\mathcal{T}_{n_{e}}\left(z_{1}\right) \overline{\mathcal{T}}_{n_{e}}^{2}\left(z_{2}\right) \mathcal{T}_{n_{e}}\left(z_{3}\right)\right\rangle \\
& =\exp \left[\frac{-\Delta_{\mathcal{T}_{n_{e}}} \mathcal{L}_{13}-\Delta_{\mathcal{T}_{\frac{n_{e}}{2}}}\left(\mathcal{L}_{12}+\mathcal{L}_{23}-\mathcal{L}_{13}\right)}{R}\right] .
\end{aligned}
$$

The holographic entanglement negativity for the mixed state configuration in question may then be expressed in terms of a specific algebraic sum of the lengths of the bulk space like geodesics as follows

$\mathcal{E}=\frac{3}{16 G_{N}^{3}}\left(\mathcal{L}_{12}+\mathcal{L}_{23}-\mathcal{L}_{13}\right)$,

where we have employed the Brown-Henneaux formula $c=$ $\frac{3 R}{2 G_{N}^{3}} \quad$ [38]. In the context of the $A d S_{d+1} / C F T_{d}$ scenario the corresponding holographic entanglement negativity for a mixed state of adjacent subsystems $A_{1}$ and $A_{2}$ in the $C F T_{d} \mathrm{~s}$ dual to bulk $A d S_{d+1}$ geometries is given as [32]

$\mathcal{E}=\frac{3}{16 G_{N}^{(d+1)}}\left(\mathcal{A}_{1}+\mathcal{A}_{2}-\mathcal{A}_{12}\right)$,

where $\mathcal{A}_{i}$ 's are the areas of co-dimension two bulk static minimal surfaces anchored on the respective subsystems $A_{i}$. It may be shown that the above expression reduces to the holographic mutual information $\mathcal{I}\left(A_{1}, A_{2}\right)$ between the two intervals (see footnote 1 ) on utilizing the Ryu-Takayanagi conjecture $\left(S_{A_{i}}=\frac{\mathcal{A}_{i}}{4 G_{N}^{(d+1)}}\right)$ as follows

$\mathcal{E}=\frac{3}{4}\left(S_{A_{1}}+S_{A_{2}}-S_{A_{1} \cup A_{2}}\right)=\frac{3}{4} \mathcal{I}\left(A_{1}, A_{2}\right)$.

We emphasize here that entanglement negativity and mutual information are completely distinct quantities in quantum information theory. Entanglement negativity provides an upper bound on the distillable entanglement of the bipartite system whereas the mutual information characterizes the total amount of correlations for the same. However, for the specific case of the adjacent subsystems our conjecture states that the holographic entanglement negativity is proportional to the holographic mutual information which is a result valid only in the holographic (large central charge) limit. Note that this does not hold for more generic bipartite configurations for example a single simply connected subsystem as described in [25] for the $A d S_{3} / C F T_{2}$ scenario and its corresponding higher dimensional generalization for the $A d S_{d+1} / C F T_{d}$ [26]. Interestingly, even for such generic configurations the holographic entanglement negativity may be expressed as certain specific algebraic sum of the holographic mutual information between appropriate subsystems. As discussed in the introduction, this relation between entanglement negativity and mutual information has also been demonstrated for time dependent scenarios involving the global and the local quenches in a $C F T_{1+1}[30,31]$. 


\section{Holographic entanglement negativity for $\mathrm{CFT}_{3}$ dual to $\mathrm{RN}_{-} \mathrm{AdS}_{4}$}

As mentioned earlier it is instructive to first examine the application of our holographic entanglement negativity conjecture to a $\mathrm{CFT}_{3}$ with a conserved charge dual to the bulk $A d S_{4}$ configurations. This exercise will elucidate the non trivial structure of the perturbative expansion for the holographic entanglement negativity for various limits of the charge and the temperature of the dual $\mathrm{CFT}_{3}$. In this context we describe the application of our conjecture to compute the holographic entanglement negativity for the bipartite mixed state configuration of adjacent subsystems with rectangular strip geometries in the $C F T_{3}$ s dual to bulk non extremal and extremal RN- $A d S_{4}$ black holes.

\subsection{Area of minimal surface for $\mathrm{RN}-A d S_{4}$ black holes}

We first briefly review the perturbative computation of the area of a co-dimension two bulk static minimal surface anchored on a subsystem of rectangular strip geometry in the dual $\mathrm{CFT}_{3}[11,33]$ which will be required for the subsequent calculations. The metric for the RN-AdS $S_{4}$ black hole with a planar horizon (with the $\operatorname{AdS}$ radius $R=1$ ) is given as

$$
\begin{aligned}
& d s^{2}=-r^{2} f(r) d t^{2}+\frac{1}{r^{2} f(r)} d r^{2}+r^{2}\left(d x^{2}+d y^{2}\right), \\
& f(r)=1-\frac{M}{r^{3}}+\frac{Q^{2}}{r^{4}} .
\end{aligned}
$$

The lapse function $f(r)$ vanishes at the horizon $\left(r=r_{h}\right)$ resulting in the following relation between the mass, charge and radius of the horizon as

$f\left(r_{h}\right)=0 \Rightarrow M=\frac{r_{h}^{4}+Q^{2}}{r_{h}}$.

One may now express the lapse function Eq. (8) in terms of the charge $Q$ and the horizon radius $r_{h}$ as follows

$f(r)=1-\frac{r_{h}^{3}}{r^{3}}-\frac{Q^{2}}{r^{3} r_{h}}+\frac{Q^{2}}{r^{4}}$.

The Hawking temperature for the $\mathrm{RN}-A d S_{4}$ black hole is given as

$T=\left.\frac{f^{\prime}(r)}{4 \pi}\right|_{r=r_{h}}=\frac{3 r_{h}}{4 \pi}\left(1-\frac{Q^{2}}{3 r_{h}^{4}}\right)$.

We now proceed to the computation the area of a codimension two static minimal surface anchored on a subsystem described by a rectangular strip geometry on the dual
$C F T_{3}$ to the RN-AdS $S_{4}$ black hole. The subsystem $A$ of rectangular strip geometry on the dual $C F T_{3}$ is specified as follows

$x \in\left[-\frac{l}{2}, \frac{l}{2}\right], \quad y \in\left[-\frac{L}{2}, \frac{L}{2}\right]$.

The area $\mathcal{A}_{A}$ of the co-dimension two bulk static minimal surface anchored on the subsystem $A$ in the holographic $C F T_{3}$ may the be expressed as

$\mathcal{A}_{A}=2 L \int_{r_{c}}^{\infty} \frac{d r}{\sqrt{f(r)\left(1-\frac{r_{c}^{4}}{r^{4}}\right)}}$.

The turning point $r_{c}$ of the minimal surface in the bulk, is related to the length of the rectangular strip in the $x$ direction as

$$
\frac{l}{2}=\int_{r_{c}}^{\infty} \frac{r_{c}^{2} d r}{r^{4} \sqrt{f(r)\left(1-\frac{r_{c}^{4}}{r^{4}}\right)}}
$$

In order to evaluate these integrals we perform a coordinate transformation from $r$ to $u=\frac{r_{c}}{r}$ and the Eqs. (10), (13) and (14) may then be expressed as

$$
\begin{aligned}
f(u) & =1-\frac{r_{h}{ }^{3} u^{3}}{r_{c}{ }^{3}}-\frac{Q^{2} u^{3}}{r_{c}{ }^{3} r_{h}}+\frac{Q^{2} u^{4}}{r_{c}{ }^{4}}, \\
\mathcal{A} & =2 L r_{c} \int_{0}^{1} \frac{f(u)^{-\frac{1}{2}}}{u^{2} \sqrt{1-u^{4}}} d u, \\
l & =\frac{2}{r_{c}} \int_{0}^{1} \frac{u^{2} f(u)^{-\frac{1}{2}}}{\sqrt{1-u^{4}}} d u .
\end{aligned}
$$

We obtain the area of the minimal surface in question through a perturbative evaluation of the above integrals for different limits of the parameters, charge $Q$ and the temperature $T$ of the $\mathrm{CFT}_{3}$.

In what follows, we compute the holographic entanglement negativity for mixed states of adjacent subsystems described by rectangular strip geometries, in $\mathrm{CFT}_{3} \mathrm{~s}$ dual to $\mathrm{RN}-A d S_{4}$ non-extremal and extremal black holes. The rectangular strip geometries corresponding to these subsystems denoted as $A_{1}$ and $A_{2}$, are specified by the coordinates:

$$
\begin{array}{ll}
x \in\left[-\frac{l_{1}}{2}, \frac{l_{1}}{2}\right], & y \in\left[-\frac{L}{2}, \frac{L}{2}\right], \\
x \in\left[-\frac{l_{2}}{2}, \frac{l_{2}}{2}\right], & y \in\left[-\frac{L}{2}, \frac{L}{2}\right],
\end{array}
$$

respectively, as depicted in Fig. 1. Note that the areas and the turning points of the corresponding co-dimension two bulk static minimal surfaces anchored on the subsystems $A_{1}$ and $A_{2}$, may therefore be obtained from Eqs. (17) and (16), by replacing $l$ in Eq. (17) by $l_{1}$ and $l_{2}$ respectively. 


\subsection{Non-extremal RN- $A d S_{4}$ black holes}

We first consider the finite temperature mixed state configuration of adjacent subsystems with rectangular strip geometries as depicted in Fig. 1, in the $C F T_{3}$ dual to a bulk nonextremal RN- $A d S_{4}$ black hole. To compute the holographic entanglement negativity utilizing our conjecture it is required to evaluate the corresponding areas of the bulk static minimal surfaces perturbatively for various limits of the relevant parameters described above.

\subsubsection{Small charge and low temperature}

The non extremality condition may be obtained in terms of the horizon radius for the bulk $\mathrm{RN}-A d S_{4}$ black hole by setting $T>0$ in Eq. (11) as follows

$r_{h}>\frac{\sqrt{Q}}{3^{\frac{1}{4}}}$

In the limit of small charge and at low temperatures it may be shown from Eq. (20) that $r_{h} \ll r_{c}$ and $Q / r_{h}^{2} \sim 1$. Hence, the function $f(u)^{-\frac{1}{2}}$ is Taylor expanded around $\frac{r_{h}}{r_{c}}=0$ to the leading order in $\mathcal{O}\left[\left(\frac{r_{h}}{r_{c}} u\right)^{3}\right]$ as follows [11]

$f(u)^{-\frac{1}{2}} \approx 1+\frac{1+\alpha}{2}\left(\frac{r_{h}}{r_{c}}\right)^{3} u^{3}$,

where $f(u)$ is the lapse function Eq. (15) for the black hole metric and $\alpha=\frac{Q^{2}}{r_{h}^{4}}$. Employing the Eqs. (21), (17) and (16), the area of the co-dimension two minimal surface anchored on the subsystem $A$ of rectangular strip geometry, may be expressed as follows [11]

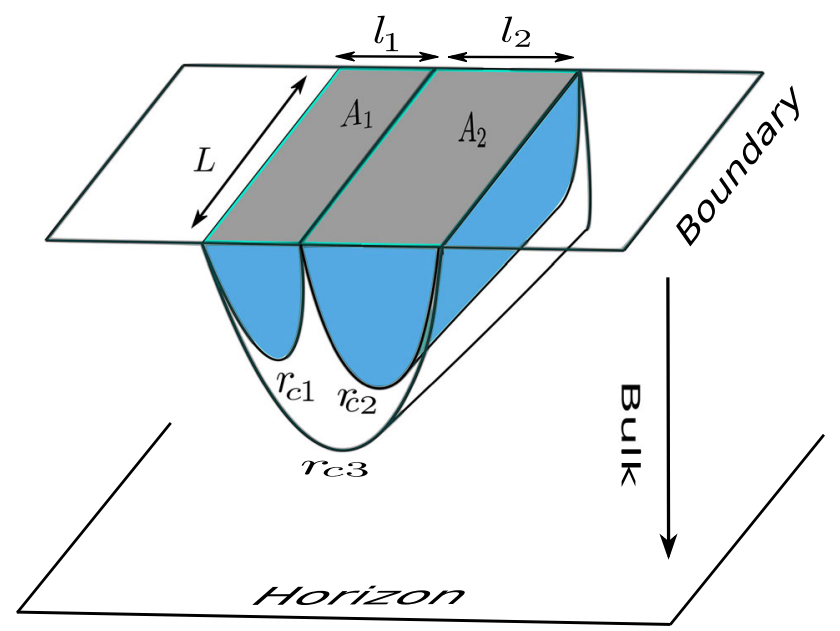

Fig. 1 Schematic of the bulk static minimal surfaces that are anchored on the subsystems $A_{1}, A_{2}$ and $A_{1} \cup A_{2}$ on the boundary $C F T_{3}$ dual to the $\mathrm{RN}-\mathrm{Ad} \mathrm{S}_{4}$ black hole
$\mathcal{A}_{A}=\mathcal{A}_{A}^{\text {div }}+\mathcal{A}_{A}^{\text {finite }}$

where the divergent part $\mathcal{A}_{A}^{\text {div }}$ and the finite part $\mathcal{A}_{A}^{\text {finite }}$ of $\mathcal{A}_{A}$ are as follows

$$
\begin{aligned}
\mathcal{A}_{A}^{\text {div }} & =2\left(\frac{L}{a}\right), \\
\mathcal{A}_{A}^{\text {finite }} & =k_{1} \frac{L}{l}+k_{2} r_{h}^{3}(1+\alpha) l^{2}+\mathcal{O}\left(r_{h}^{4} l^{3}\right) .
\end{aligned}
$$

Here the constants in the above equation are given as

$k_{1}=-\frac{4 \pi \Gamma\left(\frac{3}{4}\right)^{2}}{\Gamma\left(\frac{1}{4}\right)^{2}}$

$k_{2}=\frac{\Gamma\left(\frac{1}{4}\right)^{2}}{32 \Gamma\left(\frac{3}{4}\right)^{2}}$.

The holographic entanglement negativity in the limit of small charge and low temperature, for the mixed state of adjacent subsystems in question may now be obtained from our conjecture using Eq. (5) as follows:

$$
\begin{aligned}
\mathcal{E}= & \frac{3}{16 G_{N}^{3+1}}\left[\left(\frac{2 L}{a}\right)+k_{1}\left(\frac{L}{l_{1}}+\frac{L}{l_{2}}-\frac{L}{l_{1}+l_{2}}\right)\right. \\
& \left.-2 k_{2} M L l_{1} l_{2}\right]+\cdots,
\end{aligned}
$$

where the ellipses represent sub leading corrections in this limit. In the above equation note that the second term on the right hand side is identical to the holographic entanglement negativity for the zero temperature mixed state of adjacent subsystems in the $\mathrm{CFT}_{3}$ dual to the bulk pure $A d S_{4}$ geometry and the third term describes the correction arising from the charge and the temperature.

\subsubsection{Small charge-high temperature}

We next consider the limit of small charge and high temperature given by the conditions $r_{h} \gg 1$, and $\delta=\frac{Q}{\sqrt{3} r_{h}^{2}} \ll 1$ where as earlier $r_{h}$ represents the horizon radius. In this limit the function $f(u)^{-\frac{1}{2}}$ is Taylor expanded around $\delta=0$ as follows [11]

$$
f(u)^{-\frac{1}{2}} \approx \frac{1}{\sqrt{1-\frac{r_{h}^{3} u^{3}}{r_{c}{ }^{3}}}}+\frac{3}{2}\left(\frac{r_{h}}{r_{c}}\right)^{3} \frac{\delta^{2} u^{3}\left(1-\frac{r_{h} u}{r_{c}}\right)}{\left(1-\frac{r_{h}^{3} u^{3}}{r_{c}{ }^{3}}\right)^{3 / 2}} .
$$

Employing the above expression for the lapse function and Eqs. (17) and (16), the finite part of the area of the codimension two bulk minimal surface $\mathcal{A}_{A}$ may be expressed as

$$
\begin{aligned}
\mathcal{A}_{A}^{\text {finite }}= & L l r_{h}^{2}+\operatorname{Lr}_{h}\left(k_{1}+\delta^{2} k_{2}\right) \\
& +L r_{h} \epsilon\left[k_{3}+\delta^{2}\left(k_{4}+k_{5} \log \epsilon\right)\right]+O\left[\epsilon^{2}\right],
\end{aligned}
$$


where the constants $k_{1}, k_{2}, k_{3}, k_{4}$ and $k_{5}$ in the above equation are listed in the Appendix (A.1) in Eqs. (78), (79), (80), (81) and (82), respectively. The parameter $\epsilon$ in the Eq. (29) is given as

$\epsilon=\frac{1}{3} \exp \left(-\sqrt{3}\left(l r_{h}-c_{1}-c_{2} \delta^{2}\right)\right)$

where the constants in the above equations are listed in the Appendix (A.1) in Eqs. (83) and (84). The holographic entanglement negativity in this limit for the mixed state in question may then be computed from our conjecture as follows

$$
\begin{aligned}
\mathcal{E}= & \frac{3}{16 G_{N}^{3+1}}\left[\frac{2 L}{a}+\operatorname{Lr}_{h}\left\{\left(k_{1}+\delta^{2} k_{2}\right)+k_{3}\left(\epsilon_{1}+\epsilon_{2}-\epsilon_{12}\right)\right.\right. \\
& +\delta^{2} k_{4}\left(\epsilon_{1}+\epsilon_{2}-\epsilon_{12}\right)+\delta^{2} k_{5}\left(\log \epsilon_{1}+\log \epsilon_{2}\right. \\
& \left.\left.\left.-\log \epsilon_{12}\right)\right\}\right]+\cdots,
\end{aligned}
$$

where the subscript $i$ in $\epsilon_{i}(i=1,2,12)$ refers to the subsystems $A_{1}, A_{2}$ and $A_{1} \cup A_{2}$, respectively. Interestingly it may be noted that the holographic entanglement negativity described by the above expression depends only on the length $L$ shared between the adjacent subsystems of rectangular strip geometries (note that this is equivalent to the area of the entangling surface which in the $A d S_{4} / C F T_{3}$ scenario reduces to the length). This is unlike the holographic entanglement entropy which scales as the volume (area in the $A d S_{4} / C F T_{3}$ scenario) in this limit described in [11]. For the holographic entanglement negativity on the other hand all volume (area in $A d S_{4} / C F T_{3}$ ) dependent thermal contributions cancel leaving a purely area (length in $A d S_{4} / C F T_{3}$ ) dependent expression as expected from quantum information theory. Note that this cancellation is similar to that for the $A d S_{3} / C F T_{2}$ case described in [25,29] indicating that the elimination of the thermal contribution is possibly a universal feature of the holographic entanglement negativity in CFTs.

\subsubsection{Large charge-high temperature}

For the corresponding large charge and high temperature limit we have the conditions $r_{c} \sim r_{h}$ and $u_{0}=\frac{r_{c}}{r_{h}} \sim 1$ as a consequence of the turning point of the co dimension two static minimal surface extending close to the horizon in the bulk. In this case the Taylor expansion for the function $f(u)^{-\frac{1}{2}}$ around $u_{0}$ is given as [11]

$f(u) \approx\left(3-\frac{Q^{2}}{r_{h}^{4}}\right)\left(1-\frac{r_{h}}{r_{c}} u\right)$.
The finite part of the area of the bulk static minimal surface in this case may be expressed as

$\mathcal{A}_{A}^{\text {finite }}=L l r_{h}^{2}+\frac{L r_{h}}{2 \sqrt{\delta}}\left[K_{1}^{\prime}+K_{2}^{\prime} \epsilon+\mathcal{O}\left(\epsilon^{2}\right)\right]$,

where $\epsilon$ is given by the Eq. (30), and the constants $K_{1}^{\prime}$ and $K_{2}^{\prime}$ are listed in the Appendix (A.2) in the Eqs. (85) and (86).

The holographic entanglement negativity for the mixed state configuration of adjacent subsystems in this limit may then be obtained from our conjecture as follows

$\mathcal{E}=\frac{3}{8 G_{N}^{3+1}}\left[\left(\frac{L}{a}\right)+\frac{L r_{h}}{\sqrt{\delta}}\left\{K_{1}^{\prime}+K_{2}^{\prime}\left(\epsilon_{1}+\epsilon_{2}-\epsilon_{12}\right)\right\}\right]+\cdots$,

where the subscript $i$ in $\epsilon_{i}(i=1,2,12)$ refers to the subsystems $A_{1}, A_{2}$ and $A_{1} \cup A_{2}$ respectively. Note that as earlier the volume (area in $A d S_{4} / C F T_{3}$ ) dependent thermal terms cancel and the entanglement negativity scales as the area (length in $A d S_{4} / C F T_{3}$ ) of the entangling surface as expected from quantum information theory. Once again the elimination of thermal contribution is similar to the corresponding $A d S_{3} / C F T_{2}$ case indicating that it is an universal feature for the holographic entanglement negativity for $C F T \mathrm{~s}$.

\subsection{Extremal RN-Ad $S_{4}$ black holes}

Having described the holographic entanglement negativity for the required mixed state in the $C F T_{3}$ with a conserved charge, dual to the bulk non extremal $\mathrm{RN}-A d S_{4}$ black hole, we now turn our attention to the corresponding extremal case. To this end we consider the zero temperature mixed state configuration of adjacent subsystems with rectangular strip geometries in the $C F T_{3}$ dual to the bulk extremal RN-AdS black hole. Here we describe the computation of the holographic entanglement negativity from our conjecture, perturbatively in the limits of small and large charge.

\subsubsection{Small charge-extremal}

In the limit of small charge, the function $f(u)^{-\frac{1}{2}}$ may be Taylor expanded around $\frac{r_{h}}{r_{c}}=0$ to the leading order in $\mathcal{O}\left[\left(\frac{r_{h}}{r_{c}} u\right)^{3}\right]$ as follows [11]

$f(u)^{-\frac{1}{2}} \approx 1+2 \frac{r_{h}^{3}}{r_{c}^{3}} u^{3}$

Now employing Eqs. (35), (17) and (16) it is possible to express the finite part of the area of the bulk co-dimension two static minimal surface anchored on the subsystem $A$ as 
$\mathcal{A}_{A}^{\text {finite }}=k_{1} \frac{L}{l}+k_{2} r_{h}^{3} L l^{2}+\mathcal{O}\left(r_{h}^{4} l^{3}\right)$,

where the constants are given as follows

$$
\begin{aligned}
& k_{1}=-\frac{4 \pi \Gamma\left(\frac{3}{4}\right)^{2}}{4 \Gamma\left(\frac{1}{4}\right)^{2}}, \\
& k_{2}=\frac{4 \Gamma\left(\frac{1}{4}\right)^{2}}{32 \Gamma\left(\frac{3}{4}\right)^{2}} .
\end{aligned}
$$

The holographic entanglement negativity of the mixed state in question may then be obtained from our conjecture as follows

$$
\begin{aligned}
\mathcal{E}= & \frac{3}{16 G_{N}^{3+1}}\left[\left(\frac{2 L}{a}\right)+k_{1}\left(\frac{L}{l_{1}}+\frac{L}{l_{2}}-\frac{L}{l_{1}+l_{2}}\right)\right. \\
& \left.-2 k_{2} r_{h}^{3} L l_{1} l_{2}\right]+\cdots .
\end{aligned}
$$

The first two terms in the above equation describe the holographic entanglement negativity for the zero temperature mixed state of adjacent subsystems in the $\mathrm{CFT}_{3}$ dual to the bulk pure $A d S_{4}$ geometry and the third term describes the correction arising from the conserved charge of the extremal $\mathrm{RN}-\mathrm{Ad} \mathrm{S}_{4}$ black hole.

\subsubsection{Large charge}

As explained in the earlier sections, in the limit of large charge for the bulk extremal RN-AdS $S_{4}$ black hole we have the ratio $u_{0}=\frac{r_{c}}{r_{h}} \sim 1$. In this case the function $f(u)^{-\frac{1}{2}}$ may be Taylor expanded around $u=u_{0}$ as follows [11]

$f(u) \approx 6\left(1-\frac{r_{h}}{r_{c}} u\right)^{2}$.

Now utilizing the Eqs. (38), (17) and (16), the finite part of the area of the co dimension two static minimal surface anchored on the subsystem with rectangular strip geometry in the $C F T_{3}$ dual to the bulk extremal RN-Ad $S_{4}$ black hole in the large charge limit, may then be expressed as entropy [11]

$$
\mathcal{A}_{A}^{\text {finite }}=L l r_{h}^{2}+L r_{h}\left(K_{1}+K_{2} \sqrt{\epsilon}+K_{3} \epsilon+\mathcal{O}\left(\epsilon^{\frac{3}{2}}\right)\right) \text {, }
$$

where the constants $K_{1}, K_{2}$ and $K_{3}$ appearing in the above expression are listed in the Appendix (A.3) in the Eqs. (87), (88) and (89).

The holographic entanglement negativity for the mixed state of adjacent subsystems with rectangular strip geometries in the dual $\mathrm{CFT}_{3}$ may then be obtained from our conjecture in the large charge limit as follows:

$$
\begin{aligned}
\mathcal{E}= & \frac{3}{16 G_{N}^{3+1}}\left[\left(\frac{2 L}{a}\right)+\operatorname{Lr}_{h}\left\{K_{1}+K_{2}\left(\sqrt{\epsilon_{1}}+\sqrt{\epsilon_{2}}-\sqrt{\epsilon_{12}}\right)\right.\right. \\
& \left.\left.+K_{3}\left(\epsilon_{1}+\epsilon_{2}-\epsilon_{12}\right)\right\}\right]+\cdots
\end{aligned}
$$

where the subscripts in $\epsilon_{i}(i=1,2,12)$ refer to the subsystems $A_{1}, A_{2}$ and $A_{1} \cup A_{2}$, respectively.

Interestingly even for the extremal case in the large charge limit we once again observe that the holographic entanglement negativity for the zero temperature mixed state, following from our conjecture is purely dependent on the area (length in the $A d S_{4} / C F T_{3}$ scenario). As earlier for the non extremal case the volume (area for the $A d S_{4} / C F T_{3}$ case) dependent contributions arising from the counting entropy of the degenerate $\mathrm{CFT}_{3}$ vacuum in this case cancel leaving a purely area dependent expression as in specific earlier cases described in previous sections.

The above results for the holographic entanglement negativity of the mixed state configurations of adjacent subsystems in the dual $\mathrm{CFT}_{3}$ for various limits of the relevant parameters, conform to quantum information expectations. It is observed that in the large charge and/or large temperature regimes where the holographic entanglement entropy is dominated by volume dependent thermal contributions, the corresponding entanglement negativity depends purely on the area of the entangling surface in the $C F T$. This arises from the exact cancellation of the volume dependent thermal terms between the appropriate combinations of the contributions from the adjacent subsystems. As remarked earlier this cancellation is similar to that observed for certain $A d S_{3} / C F T_{2}$ examples and seems to be a universal feature of $C F T \mathrm{~s}$. Naturally, these results constitute strong consistency checks substantiating the higher dimensional extension of our conjecture.

Having obtained the holographic entanglement negativity for the mixed state of adjacent subsystems with rectangular strip geometries in the $A d S_{4} / C F T_{3}$ scenario and illustrating the non trivial structure of the limits associated with the perturbative expansion we now turn our attention to the generic $A d S_{d+1} / C F T_{d}$ scenario in the next section.

\section{Holographic entanglement negativity for $\mathrm{CFT}_{d}$ dual to $\mathbf{R N}-\mathbf{A d S}_{\mathbf{d}+\mathbf{1}}$}

In this section following our earlier analysis for the holographic entanglement negativity of mixed states of adjacent subsystems in the $A d S_{4} / C F T_{3}$ scenario, we now proceed to examine the corresponding case for the $A d S_{d+1} / C F T_{d}$ scenario. As earlier this case also involves a perturbative evaluation of the areas of the corresponding bulk static minimal surfaces anchored on the respective subsystems in various limits of the appropriate parameters of the RN-AdS $S_{d+1}$ black hole 
which in this case are the temperature $T$ and the chemical potential $\mu$ conjugate to the charge $Q$. Note however that for the $A d S_{d+1} / C F T_{d}$ scenario it is convenient to describe the holographic entanglement negativity in terms of an effective temperature $T_{\text {eff }}$ and another parameter $\varepsilon$ which is a function of the temperature and the chemical potential, describing the total energy of the dual $C F T_{d}$. The parameter $\varepsilon$ is therefore related to the expectation value of the $T_{00}$ component of the energy momentum tensor [37].

\subsection{Area of minimal surfaces in $\mathrm{RN}-\mathrm{AdS}_{\mathrm{d}+1}$}

The metric for the $R N-A d S_{d+1}(d \geq 3)$ black hole with the $A d S$ length scale $R=1$ is given as

$$
\begin{aligned}
d s^{2} & =\frac{1}{z^{2}}\left(-f(z) d t^{2}+\frac{d z^{2}}{f(z)}+d \mathbf{x}^{2}\right), \\
f(z) & =1-M z^{d}+\frac{(d-2) Q^{2}}{(d-1)} z^{2(d-1)}, \\
A_{t} & =Q\left(z_{H}^{d-2}-z^{d-2}\right)
\end{aligned}
$$

where $M$ and $Q$ are the mass and charge of the black hole, respectively. The location of the horizon $z_{H}$ is given by the smallest real root of the lapse function $f(z)=0$. The corresponding chemical potential $\mu$ conjugate to the charge $Q$ is defined as follows:

$\mu \equiv \lim _{z \rightarrow 0} A_{t}(z)=Q z_{H}^{d-2}$

and the Hawking temperature is

$T=-\left.\frac{1}{4 \pi} \frac{d}{d z} f(z)\right|_{z_{H}}=\frac{d}{4 \pi z_{H}}\left(1-\frac{(d-2)^{2} Q^{2} z_{H}^{2(d-1)}}{d(d-1)}\right)$.

The lapse function, chemical potential and the temperature may now be expressed as follows:

$$
\begin{aligned}
f(z) & =1-\varepsilon\left(\frac{z}{z_{H}}\right)^{d}+(\varepsilon-1)\left(\frac{z}{z_{H}}\right)^{2(d-1)}, \\
\mu & =\frac{1}{z_{H}} \sqrt{\frac{(d-1)}{(d-2)}(\varepsilon-1),} \\
T & =\frac{2(d-1)-(d-2) \varepsilon}{4 \pi z_{H}} .
\end{aligned}
$$

Here $\varepsilon$ is a dimensionless quantity with limits $1 \geq \varepsilon \geq$ $\frac{2(d-1)}{d-2}$, that describes the energy of the system [37], as follows

$$
\varepsilon(T, \mu)=b_{0}-\frac{2 n}{1+\sqrt{1+\frac{d^{2}}{2 \pi^{2} b_{0} b_{1}}\left(\frac{\mu^{2}}{T^{2}}\right)}},
$$

where the constants $b_{0}$ and $b_{1}$ are given as

$b_{0}=\frac{2(d-1)}{d-2}, \quad b_{1}=\frac{d}{d-2}$.

The effective temperature $T_{\text {eff }}$ describing the number of microstates for a given temperature and chemical potential may be defined as [37]

$T_{\text {eff }}(T, \mu) \equiv \frac{d}{4 \pi z_{H}}=\frac{T}{2}\left[1+\sqrt{1+\frac{d^{2}}{2 \pi^{2} b_{0} b_{1}}\left(\frac{\mu^{2}}{T^{2}}\right)}\right]$.

We now proceed to compute the area of a co dimension two bulk static minimal surface anchored on a subsystem with rectangular strip geometry in the dual $C F T_{d}$. The strip geometry of the subsystem in question may then be specified as follows

$x \equiv x^{1} \in\left[-\frac{l}{2}, \frac{l}{2}\right], x^{i} \in\left[-\frac{L}{2}, \frac{L}{2}\right], \quad i=2, \ldots, d-2$,

where $L \rightarrow \infty$. The area $\mathcal{A}$ of the co-dimension two bulk extremal surface anchored on the subsystem in the boundary may be expressed as

$$
\begin{aligned}
\mathcal{A} & =2 L^{d-2} z_{*}^{d-1} \int_{0}^{l / 2} \frac{d x}{z(x)^{2(d-1)}} \\
& =2 L^{d-2} z_{*}^{d-1} \int_{a}^{z_{*}} \frac{d z}{z^{d-1} \sqrt{f(z)\left[z_{*}^{2(d-1)}-z^{2(d-1)}\right]}},
\end{aligned}
$$

where $a$ is the UV cut off of the $C F T_{d}$. The turning point $z_{*}$ of the extremal surface in the bulk is related to $l$, the length of the strip in the $x^{1}$ direction as

$\frac{l}{2}=\int_{0}^{z_{*}} \frac{d z}{\sqrt{f(z)\left[\left(z_{*} / z\right)^{2(d-1)}-1\right]}}$.

The authors in [37] demonstrated that the above integral may be expressed as a double sum as

$$
\begin{aligned}
l= & \frac{z_{*}}{d-1} \sum_{n=0}^{\infty} \sum_{k=0}^{n} \frac{\Gamma\left[\frac{1}{2}+n\right] \Gamma\left[\frac{d(n+k+1)-2 k}{2(d-1)}\right] \varepsilon^{n-k}(1-\varepsilon)^{k}}{\Gamma[1+n-k] \Gamma[k+1] \Gamma\left[\frac{d(n+k+2)-2 k-1}{2(d-1)}\right]} \\
& \times\left(\frac{z_{*}}{z_{H}}\right)^{n d+k(d-2)}
\end{aligned}
$$

The area of the static minimal surface may also be expressed as a double sum as follows [37]

$$
\mathcal{A}=\frac{2}{d-2}\left(\frac{L}{a}\right)^{d-2}+2 \frac{L^{d-2}}{z_{*}^{d-2}}\left[\frac{\sqrt{\pi} \Gamma\left(-\frac{d-2}{2(d-1)}\right)}{2(d-1) \Gamma\left(\frac{1}{2(d-1)}\right)}\right]
$$




$$
\begin{aligned}
& +\frac{L^{d-2}}{(d-1) z_{*}^{d-2}}\left[\sum_{n=1}^{\infty} \sum_{k=0}^{n} \frac{\Gamma\left[\frac{1}{2}+n\right] \Gamma\left[\frac{d(n+k-1)-2 k+2}{2(d-1)}\right] \varepsilon^{n-k}(1-\varepsilon)^{k}}{\Gamma[1+n-k] \Gamma[k+1] \Gamma\left[\frac{d(n+k)-2 k+1}{2(d-1)}\right]}\right. \\
& \left.\times\left(\frac{z_{*}}{z_{H}}\right)^{n d+k(d-2)}\right] .
\end{aligned}
$$

The area and the turning point of the corresponding static minimal surface are expressed in terms of the specified parameters $T_{\text {eff }}$ and $\varepsilon$ as a perturbation expansion, for various limits of the chemical potential $\mu$ and the temperature $T$ of the dual $C F T_{d}$. We now proceed to describe these evaluations and utilize them to obtain the holographic entanglement negativity for the mixed states in question, from our conjecture.

In what follows, we compute the holographic entanglement negativity for mixed states of adjacent subsystems described by rectangular strip geometries in $C F T_{d} \mathrm{~s}$ dual to $\mathrm{RN}-A d S_{d+1}$ non-extremal and extremal black holes. The rectangular strip geometries corresponding to these subsystems denoted as $A_{1}$ and $A_{2}$, are specified by the coordinates

$$
\begin{aligned}
& x^{1} \in\left[-\frac{l_{1}}{2}, \frac{l_{1}}{2}\right], \quad x^{i} \in\left[-\frac{L}{2}, \frac{L}{2}\right], \\
& x^{1} \in\left[-\frac{l_{2}}{2}, \frac{l_{2}}{2}\right], \quad x^{i} \in\left[-\frac{L}{2}, \frac{L}{2}\right],
\end{aligned}
$$

respectively, as depicted in Fig. (1) (with $L$ now denoting the length of the strip in the remaining $(d-2)$ directions). Note that the areas and the turning points of the corresponding co-dimension two bulk static minimal surfaces anchored on the subsystems $A_{1}$ and $A_{2}$, may therefore be obtained from Eqs. (51) and (52), by replacing $l$ in Eq. (52) by $l_{1}$ and $l_{2}$, respectively.

\subsection{Non-extremal RN-AdS $\mathrm{S}_{\mathrm{d}+1}$}

We first consider the non-extremal $R N-A d S_{d+1}$ black holes and compute the holographic entanglement negativity for the finite temperature mixed state of adjacent subsystems described by rectangular strip geometries in the dual $C F T_{d}$ fr various limits of the chemical potential $\mu$ and the temperature $T$.

\subsubsection{Small chemical potential_-low temperature}

The limit of small chemical potential and low temperature is defined by the conditions $T l \ll 1$ and $\mu l \ll 1$. Notice that apart from the chemical potential $\mu$ and the temperature $T$, the area of the static minimal surface depends on the length of the rectangular strip along the $x^{1}$ direction provided we keep the lengths in all the other $x^{i}$ direction to be the constant $L$. Hence, the limit of small chemical potential and low temperature has to be fixed by specifying another condition which is chosen to be $T \ll \mu$ or $T \gg \mu$ as described in [37].
Below we compute the holographic entanglement negativity of the required mixed state for both of the above mentioned limits.

(i) $T l \ll \mu l \ll 1$

We first consider the limit defined by the conditions $T l \ll 1$, $\mu l \ll 1$ and $T \ll \mu$ which may be re-casted as $T l \ll \mu l \ll$ 1. In the limit $T \ll \mu$, the parameters $T_{\text {eff }}(T, \mu)$ and $\varepsilon(T, \mu)$ described by Eqs. (49) and (47) may be approximated by Taylor expanding them around $\frac{T}{\mu}=0$ to the leading order as follows [37]

$$
\begin{aligned}
T_{\mathrm{eff}} & \approx \frac{1}{2}\left(\frac{\mu d}{\pi \sqrt{2 b_{0} b_{1}}}+T\right), \\
\varepsilon & \approx b_{0}-\frac{2 n \pi \sqrt{2 b_{0} b_{1}}}{d}\left(\frac{T}{\mu}\right) .
\end{aligned}
$$

Now from the other two conditions $T l \ll 1$ and $\mu l \ll 1$ it may be shown that the turning point of the static minimal surface is far from the horizon i.e., $z_{*} \ll z_{H}$. Hence the expression for the turning point may be obtained by expanding Eq. (53) to the leading order in $\left(\frac{l}{z_{H}}\right)^{d}$ as

$$
\begin{aligned}
z_{*}= & \frac{l \Gamma\left[\frac{1}{2(d-1)}\right]}{2 \sqrt{\pi} \Gamma\left[\frac{d}{2(d-1)}\right]} \\
& \times\left[1-\frac{1}{2(d+1)} \frac{2^{\frac{1}{d-1}-d} \Gamma\left(1+\frac{1}{2(d-1)}\right) \Gamma\left(\frac{1}{2(d-1)}\right)^{d+1}}{\pi^{\frac{d+1}{2}} \Gamma\left(\frac{1}{2}+\frac{1}{d-1}\right) \Gamma\left(\frac{d}{2(d-1)}\right)^{d}}\right. \\
& \left.\varepsilon\left(\frac{l}{z_{H}}\right)^{d}+\mathcal{O}\left(\frac{l}{z_{H}}\right)^{2(d-1)}\right] .
\end{aligned}
$$

Similarly, the area of the minimal surface may be obtained by Eq. (54) to the leading order in $\left(\frac{l}{z_{H}}\right)^{d}$ and is re-expressed in terms of $T_{\text {eff }}$ and $\varepsilon$ as follows [37]

$$
\begin{aligned}
\mathcal{A}_{A}= & {\left[\frac{2}{d-2}\left(\frac{L}{a}\right)^{d-2}+\mathcal{S}_{0}\left(\frac{L}{l}\right)^{d-2}\right.} \\
& \left.+\varepsilon \mathcal{S}_{0} \mathcal{S}_{1}\left(\frac{4 \pi T_{\mathrm{eff}}}{d}\right)^{d} L^{d-2} l^{2}\right]+\mathcal{O}\left(T_{\mathrm{eff}} l\right)^{2(d-1)} .
\end{aligned}
$$

The holographic entanglement negativity for the mixed state in question, is then given as

$$
\begin{aligned}
\mathcal{E}= & \frac{3}{16 G_{N}^{d+1}}\left[\frac{2}{d-2}\left(\frac{L}{a}\right)^{d-2}\right. \\
& +\mathcal{S}_{0} L^{d-2}\left(\frac{1}{l_{1}^{d-2}}+\frac{1}{l_{2}^{d-2}}-\frac{1}{\left(l_{1}+l_{2}\right)^{d-2}}\right) \\
& \left.-\varepsilon \mathcal{S}_{0} \mathcal{S}_{1}\left(\frac{4 \pi T_{\mathrm{eff}}}{d}\right)^{d} L^{d-2} 2 l_{1} l_{2}\right]+\cdots
\end{aligned}
$$

In the above expression for the holographic entanglement negativity for the finite temperature mixed state the first two 
terms are identical to those in the holographic negativity for the zero temperature mixed state of adjacent subsystems in the $C F T_{d}$ which is dual to the bulk pure $A d S_{d+1}$ geometry. The other term describes the correction due to the chemical potential and the temperature of the black hole.

\section{(ii) $\mu l \ll T l \ll 1$}

We consider the limit $T l \ll 1, \mu l \ll 1$ and $T \gg \mu$ which may be recast as $\mu l \ll T l \ll 1$. In this limit the parameters $T_{\text {eff }}(T, \mu)$ and $\varepsilon(T, \mu)$ described by Eqs. (49) and (47) may be Taylor expanded around $\frac{\mu}{T}=0$ to the leading order as follows [37]

$$
\begin{aligned}
& T_{\mathrm{eff}}=T\left[1+\frac{d(d-2)^{2}}{16 \pi^{2}(d-1)}\left(\frac{\mu}{T}\right)^{2}+\mathcal{O}\left(\frac{\mu}{T}\right)^{4}\right], \\
& \varepsilon=1+\frac{d^{2}(d-2)}{16 \pi^{2}(d-1)}\left(\frac{\mu}{T}\right)^{2}+\mathcal{O}\left(\frac{\mu}{T}\right)^{4} .
\end{aligned}
$$

Once again from the conditions $T l \ll 1$ and $\mu l \ll 1$ it is clear that $z_{*} \ll z_{H}$. The expressions for the turning point may be obtained by expanding Eq. (53) to the leading order in $\left(\frac{l}{z_{H}}\right)^{d}$ and is same as the one given in Eq. (59). The area of the co dimension two minimal surface anchored on the subsystem $A$ of rectangular strip geometry in the dual $C F T_{d}$, is once again determined by expanding Eq. (54) to the leading order in $\left(\frac{l}{z_{H}}\right)^{d}$ as: [37]

$$
\begin{aligned}
\mathcal{A}_{A}= & {\left[\frac{2}{d-2}\left(\frac{L}{a}\right)^{d-2}+\mathcal{S}_{0}\left(\frac{L}{l}\right)^{d-2}\right.} \\
& \left.+\varepsilon \mathcal{S}_{0} \mathcal{S}_{1}\left(\frac{4 \pi T_{\mathrm{eff}}}{d}\right)^{d} L^{d-2} l^{2}\right]+\mathcal{O}\left(T_{\mathrm{eff}} l\right)^{2(d-1)},
\end{aligned}
$$

where the numerical constants $\mathcal{S}_{0}$ and $\mathcal{S}_{1}$ are listed in the Appendix (B.1) in the Eqs. (90) and (91), respectively. Note that although the area of the static minimal surface given in Eqs. (60) and (64) have identical forms for both $T l \ll$ $\mu l \ll 1$ and $\mu l \ll T l \ll 1$, the expressions for the effective temperature $T_{\text {eff }}$ and the parameter $\varepsilon$, for this case are distinct as given by Eqs. (62) and (63), respectively.

The holographic entanglement negativity for the required finite temperature mixed state of adjacent subsystems with rectangular strip geometries, in the limit of small charge and low temperature may then be given from our conjecture as follows:

$$
\begin{aligned}
\mathcal{E}= & \frac{3}{16 G_{N}^{d+1}}\left[\frac{2}{d-2}\left(\frac{L}{a}\right)^{d-2}\right. \\
& +\mathcal{S}_{0} L^{d-2}\left(\frac{1}{l_{1}^{d-2}}+\frac{1}{l_{2}^{d-2}}-\frac{1}{\left(l_{1}+l_{2}\right)^{d-2}}\right) \\
& \left.-\varepsilon \mathcal{S}_{0} \mathcal{S}_{1}\left(\frac{4 \pi T_{\mathrm{eff}}}{d}\right)^{d} L^{d-2} 2 l_{1} l_{2}\right]+\cdots
\end{aligned}
$$

Once again it may observed that the holographic entanglement negativity for the finite temperature mixed state contains three terms. The first two terms are identical to the holographic negativity for the zero temperature mixed state of adjacent subsystems in the $C F T_{d}$ which is dual to the bulk pure $A d S_{d+1}$ geometry. The third term describes the correction due to the chemical potential and the temperature of the black hole.

\subsubsection{Small chemical potential_-high temperature}

Having computed the holographic entanglement for the mixed state in question in the limit of small chemical and low temperature, we now proceed to obtain the same in the limit of small chemical potential and high temperature. This limit is defined by the conditions $\mu \ll T$ and $T l \gg 1$ as described in [37]. As explained in the previous subsection for $\mu \ll T$, the parameter $T_{\text {eff }}$ and $\varepsilon$ may be approximated by Taylor expanding them around $\frac{\mu}{T}=0$, to the leading order in $\frac{\mu}{T}$ as given by Eqs. (62) and (63). However, in contrast to the previous case, the other condition $T l \gg 1$ implies that the turning point of the minimal surface is close to the horizon i.e., $z_{*} \sim z_{H}$. Hence, the area of the static minimal surface may be obtained perturbatively from Eqs. (54) by expanding it around $\frac{z_{*}}{z_{H}}=1$ as follows

$$
\begin{aligned}
\mathcal{A}_{A}= & {\left[\frac{2}{d-2}\left(\frac{L}{a}\right)^{d-2}+V\left(\frac{4 \pi T_{\mathrm{eff}}}{d}\right)^{d-1}\right.} \\
& \left.+L^{d-2}\left(\frac{4 \pi T_{\mathrm{eff}}}{d}\right)^{d-2} \gamma_{d}\left(\frac{\mu}{T}\right)\right],
\end{aligned}
$$

where $V=L^{d-2} l$ is the volume of the strip, and the function $\gamma_{d}\left(\frac{\mu}{T}\right)$ in the above expression is perturbative in $\frac{\mu}{T}$ as given in the Appendix (B.2) in the Eq. (92).

The holographic entanglement negativity for the mixed state in question may then be obtained from our conjecture as follows

$$
\begin{aligned}
\mathcal{E}= & \frac{3}{16 G_{N}^{d+1}}\left[\frac{2}{d-2}\left(\frac{L}{a}\right)^{d-2}\right. \\
& \left.+L^{d-2}\left(\frac{4 \pi T_{\mathrm{eff}}}{d}\right)^{d-2} \gamma_{d}\left(\frac{\mu}{T}\right)\right] .
\end{aligned}
$$

Note that in the above equation the leading contribution to the holographic entanglement negativity for the mixed state in this limit is purely dependent on the area of the entangling surface between the adjacent subsystems with rectangular strip geometries. As earlier for the $A d S_{4} / C F T_{3}$ case the volume dependent thermal contributions cancel leaving an expression that is purely area dependent. This once again conforms to the standard quantum information expectations for the negativity and serves as a strong consistency check for our conjecture. 


\subsubsection{Large chemical potential-low temperature}

The limit of large chemical potential and low temperature is described by the conditions $\mu l \gg 1$ and $T \ll \mu$. As explained earlier utilizing the condition $T \ll \mu$, the parameters $T_{\text {eff }}(T, \mu)$ and $\varepsilon(T, \mu)$ may be approximated by Taylor expanding them around $\frac{T}{\mu}=0$ as given by Eqs. (57) and (58), respectively. Employing the other condition $\mu l \gg 1$ implies that the turning point of the minimal surface is close to the horizon i.e., $z_{*} \sim z_{H}$. Hence, the area of the static minimal surface may once again be obtained perturbatively from Eqs. (54) by expanding it around $\frac{z_{*}}{z_{H}}=1$ as follows [37]

$$
\begin{aligned}
\mathcal{A}_{A}= & {\left[\frac{2}{d-2}\left(\frac{L}{a}\right)^{d-2}+V\left(\frac{4 \pi T_{\text {eff }}}{d}\right)^{d-1}\right.} \\
& +L^{d-2}\left(\frac{4 \pi T_{\text {eff }}}{d}\right)^{d-2}\left(N_{0}+N_{1}\left(b_{0}-\varepsilon\right)\right) \\
& \left.+\mathcal{O}\left(\frac{T}{\mu}\right)\right],
\end{aligned}
$$

where $V=L^{d-2} l$ is the volume of the strip, and the numerical constant in the above expression $N_{0}$ and $N_{1}$ are listed in the Appendix (B.3) in the Eqs. (94) and (95), respectively.

The holographic entanglement negativity for the finite temperature mixed state of adjacent subsystem in question may then be obtained from our conjecture as follows

$$
\begin{aligned}
\mathcal{E}= & \frac{3}{16 G_{N}^{d+1}}\left[\frac{2}{d-2}\left(\frac{L}{a}\right)^{d-2}\right. \\
& \left.+L^{d-2}\left(\frac{4 \pi T_{\mathrm{eff}}}{d}\right)^{d-2}\left(N_{0}+N_{1}\left(b_{0}-\varepsilon\right)\right)\right]+\cdots .
\end{aligned}
$$

We observe from the above expression that in the limit of large chemical potential and low temperature, the holographic entanglement negativity obtained from our conjecture is purely dependent on the area of the entangling surface between the adjacent subsystems with rectangular strip geometries. As earlier this indicates the cancellation of the volume dependent thermal contributions conforming to the usual quantum information theory expectations and constitutes yet another fairly strong consistency check for our conjecture.

\subsection{Extremal $\mathrm{RN}-\mathrm{AdS}_{\mathrm{d}+1}$}

Having obtained the holographic entanglement negativity for the finite temperature mixed state in the $C F T_{d}$ dual to the bulk non extremal RN- $A d S_{d+1}$ black hole, we now turn our attention to the zero temperature mixed state dual to a bulk extremal RN-Ad $S_{d+1}$ black hole. The relevant parameters in this case are given as [37]

$$
\begin{aligned}
Q^{2} & =d(d-1) L^{2} /(d-2)^{2} z_{H}^{2(d-1)}, \\
\varepsilon & =b_{1}, \\
\mu & =\frac{1}{z_{H}} \sqrt{\frac{b_{0} b_{1}}{2}}=\frac{1}{z_{H}} \sqrt{\frac{d(d-1)}{(d-2)^{2}}}, \\
T_{\mathrm{eff}} & =\frac{\mu d}{2 \pi \sqrt{2 b_{0} b_{1}}} .
\end{aligned}
$$

Here $Q$ represents the charge of the extremal RN-AdS $S_{d+1}$ black hole and $T_{e f f}$ is the effective temperature as earlier. Using the parameters as given above we now proceed to obtain the area of a co-dimension two bulk minimal surface anchored on a subsystem with rectangular strip geometry in a perturbative expansion for various limits of the charge $Q$. The area expression may then be utilized to obtain the holographic entanglement negativity for the mixed state in question from our conjecture.

\subsubsection{Small chemical potential}

Note that in the small chemical potential limit defined by the condition $\mu l \ll 1$ the turning point of the static minimal surface is far away from the horizon $z_{*} \ll z_{H}$. Hence the Eq. (52) may be solved for $z_{*}$ and at leading order in $\left(l / z_{H}\right)^{d}$ which once again leads to Eq. (59) [37]. The area of the minimal surface anchored on the subsystem- $A$ of rectangular strip geometry may then obtained perturbatively expanding Eq. (54) to the leading order in $\left(l / z_{H}\right)^{d}$. Upon re-expressing the area of the static minimal surface in terms of $\mu$, it is possible to show that [37]

$$
\begin{aligned}
\mathcal{A}_{A}= & {\left[\frac{2}{d-2}\left(\frac{L}{a}\right)^{d-2}+\mathcal{S}_{0}\left(\frac{L}{l}\right)^{d-2}\right.} \\
& +\mathcal{S}_{0} \mathcal{S}_{1} \frac{2(d-1)}{d-2}\left(\frac{(d-2) \mu}{\sqrt{d(d-1)}}\right)^{d} L^{d-2} l^{2} \\
& \left.+\mathcal{O}\left[(\mu l)^{2(d-1)}\right]\right]
\end{aligned}
$$

where the constants $\mathcal{S}_{0}, \mathcal{S}_{1}$ are identical to earlier cases and given in the Appendix. The holographic entanglement negativity for the mixed state of the adjacent subsystem of rectangular strip geometries, in the small charge limit may then be obtained utilizing our conjecture as follows

$$
\begin{aligned}
\mathcal{E}= & \frac{3}{16 G_{N}^{d+1}}\left[\frac{2}{d-2}\left(\frac{L}{a}\right)^{d-2}\right. \\
& +\mathcal{S}_{0} L^{d-2}\left(\frac{1}{l_{1}^{d-2}}+\frac{1}{l_{2}^{d-2}}-\frac{1}{\left(l_{1}+l_{2}\right)^{d-2}}\right) \\
& \left.-\mathcal{S}_{0} \mathcal{S}_{1} \frac{2(d-1)}{d-2}\left(\frac{(d-2) \mu}{\sqrt{d(d-1)}}\right)^{d} L^{d-2} 2 l_{1} l_{2}\right]+\cdots
\end{aligned}
$$


Observe that the first two terms in the above expression correspond to the holographic entanglement negativity for the zero temperature mixed state of adjacent subsystems with rectangular strip geometries in the $C F T_{d}$ dual to the bulk pure $A d S_{d+1}$ geometry. The other term along with the sub leading higher order terms describe the correction due to the chemical potential of the $C F T_{d}$.

\subsubsection{Large chemical potential}

For the case of extremal $R N-A d S_{d+1}$ black hole, the limit of the large chemical potential is specified by the condition $\mu l \gg 1$. Hence, it may be observed from Eq. (70) that the horizon radius is large and the turning point of the static minimal surface is therefore close to the horizon i.e., $z_{*} \rightarrow$ $z_{H}$. The area of the static minimal surface anchored on the subsystem- $A$ of rectangular strip geometry may be obtained by evaluating the integral in Eq. (54) perturbatively around $z_{*} / z_{H}=1$ as follows

$$
\begin{aligned}
\mathcal{A}_{A}= & {\left[\frac{2}{d-2}\left(\frac{L}{a}\right)^{d-2}+V \mu^{d-1}\left(\frac{d-2}{\sqrt{d(d-1)}}\right)^{d-1}\right.} \\
& \left.+L^{d-2} N\left(b_{0}\right)\left(\frac{d-2}{\sqrt{d(d-1)}}\right)^{d-2} \mu^{d-2}\right],
\end{aligned}
$$

where $V=L^{d-2} l$ is the volume of the strip and $N\left(b_{0}\right)$ is the value of $N(\varepsilon)$ at $\varepsilon=b_{0}$. The holographic entanglement negativity for the mixed state of the adjacent subsystem of rectangular strip geometries, in the large charge limit may then be obtained utilizing our conjecture as follows

$$
\begin{aligned}
\mathcal{E}= & \frac{3}{16 G_{N}^{d+1}}\left[\frac{2}{d-2}\left(\frac{L}{a}\right)^{d-2}\right. \\
& \left.+L^{d-2} N\left(b_{0}\right)\left(\frac{d-2}{\sqrt{d(d-1)}}\right)^{d-2} \mu^{d-2}\right] .
\end{aligned}
$$

We observe from the above equation that in the limit of large chemical potential also the holographic entanglement negativity obtained from our conjecture is purely dependent on the area of the entangling surface shared by the adjacent subsystems with rectangular strip geometries. As earlier for the extremal case in the $A d S_{4} / C F T_{3}$ scenario, the volume dependent contributions arising from the counting entropy of the degenerate $C F T_{d}$ vacuum, cancel leaving a purely area dependent expression in conformity with quantum information expectation.

\section{Summary and conclusion}

To summarize, we have applied our holographic entanglement negativity conjecture for bipartite mixed state configurations of adjacent subsystems to specific examples of
$C F T_{d}$ s dual to bulk non extremal and extremal RN- $A d S_{d+1}$ black holes. Our conjecture involves a specific algebraic sum of the areas of co-dimension two bulk static minimal surfaces anchored on the appropriate subsystems in the dual $C F T_{d}$. In this context we have considered mixed state configurations of adjacent subsystems with rectangular strip geometries in the holographic $C F T_{d}$.

In this exercise we have first studied the above examples in the $A d S_{4} / C F T_{3}$ scenario to elucidate the non trivial structure of the perturbative expansion for the holographic entanglement negativity involving various limits of the relevant parameters. For the finite temperature mixed states of adjacent subsystems in the $C F T_{3}$ dual to bulk non extremal $\mathrm{RN}-A d S_{3+1}$ black hole, we observe the following behavior for the holographic entanglement negativity. In the small charge and low temperature limit the leading part of the holographic entanglement negativity includes a contribution from the zero temperature mixed state of adjacent subsystems in the $C F T_{3}$ which is dual to the bulk pure $A d S_{4}$ geometry and a correction term involving the charge and the temperature. This is because in this limit the bulk static minimal surfaces are located far away from the black hole horizon and the leading contribution to the holographic entanglement negativity arises from the near boundary pure $A d S_{4}$ geometry. On the other hand in the limits of large charge and low temperature and vice versa the leading part of the holographic entanglement negativity depends purely on the area of the entangling surface (length in $A d S_{4} / C F T_{3}$ ) and the volume (area in $A d S_{4} / C F T_{3}$ ) dependent thermal terms cancel. This is in conformity with quantum information expectation for the entanglement negativity, as the dominant contribution arises from the entanglement between the degrees of freedom at the entangling surface (line for the $A d S_{4} / C F T_{3}$ scenario) shared between the adjacent subsystems. For the case of the zero temperature mixed state of the $C F T_{3}$ dual to the bulk extremal RN- $A d S_{3+1}$ black hole, the leading contribution to the holographic entanglement negativity in the small charge limit consists of two parts. These involve the contribution from the zero temperature mixed state in the $C F T_{3}$ dual to the bulk pure $A d S_{4}$ geometry and a correction term involving the charge. In contrast, in the limit of large charge the leading part of the holographic entanglement negativity depends only on the area of the entangling surface (length in $A d S_{4} / C F T_{3}$ ). This is due to the cancellation of the volume (area in $A d S_{4} / C F T_{3}$ ) dependent terms arising from the counting entropy of the degenerate $C F T_{3}$ vacuum.

Following the above exercise for the $A d S_{4} / C F T_{3}$ scenario to clarify the non trivial limits associated with the perturbation expansion, we have subsequently described the holographic entanglement negativity in the general $A d S_{d+1} / C F T_{d}$ case. To this end the relevant perturbative expansion of the holographic entanglement negativity requires the introduction of distinct parameters described by 
the energy and an effective temperature of the $C F T_{d}$. The leading contribution to the holographic entanglement negativity following from our conjecture exhibits identical behavior to that of the corresponding $A d S_{4} / C F T_{3}$ case for the appropriate limits of the relevant parameters.

Our results for the applications described above, conform to the standard quantum information expectations. This may be observed from the fact that for the small chemical potential and low temperature, the contribution to the holographic entanglement negativity arises from that of the zero temperature mixed state of the $C F T_{d}$ dual to the bulk pure $A d S_{d+1}$ geometry and corrections due to the chemical potential and the temperature. This conforms to the fact that in this limit the mixed state in question of the $C F T_{d}$ dual to the non extremal bulk RN- $A d S_{d+1}$ is dominated by the quantum correlations. For the extremal black hole on the other hand, in this limit the holographic entanglement negativity arises from that of a distinct zero temperature mixed state of the $C F T_{d}$ dual to the bulk pure $A d S_{d+1}$ geometry (this mixed state is obtained by tracing over the pure vacuum state of the $C F T_{d}$ ) and corrections due to the chemical potential. Once again this indicates that the mixed state above is dominated by the quantum correlations in this limit.

Furthermore our results also demonstrate the exact cancellation of the volume dependent thermal terms for the holographic entanglement negativity of the mixed state in the limit of large chemical potential and/or high temperature. Interestingly for the zero temperature mixed state of the $C F T_{d}$ dual to the extremal RN- $A d S_{d+1}$ black holes the cancellation involves the volume dependent counting entropy of the corresponding degenerate $C F T_{d}$ vacuum. Our results seemingly indicates that this is a universal feature of the holographic entanglement negativity for $C F T \mathrm{~s}$. In both these cases the holographic entanglement negativity depends purely on the area of the entangling surface shared between the adjacent subsystems in conformity with quantum information theory expectations.

These constitute important consistency checks for the universality of our conjecture which should find interesting applications in diverse areas such as condensed matter physics and issues of quantum gravity. We should however mention here that a bulk proof for our conjecture along the lines of [17] is a critical open issue which needs attention. Note also that our conjecture is applicable to the specific mixed state configuration involving adjacent subsystems only. The more general case involving the mixed state of disjoint subsystems remains an interesting open problem which is not expected to be a straightforward generalization of the construction described here. This is because the corresponding $C F T_{1+1}$ results obtained through the replica technique requires the evaluation of a four point twist correlator which involves a non universal arbitrary function. Very recently we have addressed this issue in [39] where we have proposed a holographic construction for the mixed state configuration of two disjoint intervals in a dual $C F T_{1+1}$ and substantiated this with specific examples. We hope to address other related fascinating issues and further applications in the near future.

Acknowledgements Parul Jain would like to thank Prof. Mariano Cadoni for his guidance and the Department of Physics, Indian Institute of Technology Kanpur, India for their warm hospitality. Parul Jain's work is financially supported by Università di Cagliari, Italy and INFN, Sezione di Cagliari, Italy.

Open Access This article is distributed under the terms of the Creative Commons Attribution 4.0 International License (http://creativecomm ons.org/licenses/by/4.0/), which permits unrestricted use, distribution, and reproduction in any medium, provided you give appropriate credit to the original author(s) and the source, provide a link to the Creative Commons license, and indicate if changes were made.

Funded by SCOAP . $^{3}$

\section{Appendix A: Non-extremal and extremal RN-AdS 4}

\author{
A.1 Non-extremal RN- $A d S_{4}$ (Small charge - high \\ temperature)
}

The constants $k_{1}, k_{2}, k_{3}, k_{4}$ and $k_{5}$ in the Eq. (29) are given as follows

$$
\begin{aligned}
k_{1}= & \sum_{n=1}^{\infty}\left(\frac{1}{3 n-1} \frac{\Gamma\left(n+\frac{1}{2}\right)}{\Gamma(n+1)} \frac{\Gamma\left(\frac{3 n+3}{4}\right)}{\Gamma\left(\frac{3 n+5}{4}\right)}-\frac{2}{3 \sqrt{3} n^{2}}\right) \\
& +\frac{\pi^{2}}{9 \sqrt{3}}+\frac{\sqrt{\pi} \Gamma\left(-\frac{1}{4}\right)}{\Gamma\left(\frac{1}{4}\right)}, \\
k_{2}= & \frac{3 \pi}{8}-\frac{3 \Gamma\left(\frac{3}{2}\right) \Gamma\left(\frac{7}{4}\right)}{\Gamma\left(\frac{9}{4}\right)} \\
& +3 \sum_{n=1}^{\infty}\left(\frac{1}{3 n+2} \frac{\Gamma\left(n+\frac{3}{2}\right)}{\Gamma(n+1)} \frac{\Gamma\left(\frac{3 n+6}{4}\right)}{\Gamma\left(\frac{3 n+8}{4}\right)}-\frac{1}{3 \sqrt{3} n}\right) \\
& -3 \sum_{n=1}^{\infty}\left(\frac{2}{3 n+3} \frac{\Gamma\left(n+\frac{3}{2}\right)}{\Gamma(n+1)} \frac{\Gamma\left(\frac{3 n+7}{4}\right)}{\Gamma\left(\frac{3 n+9}{4}\right)}-\frac{2}{3 \sqrt{3} n}\right), \\
k_{3}= & \frac{-2}{\sqrt{3}}+\frac{\pi^{2}}{9 \sqrt{3}}, \\
k_{4}= & \frac{2}{\sqrt{3}}-\frac{2}{\sqrt{3}} \log [3]+\frac{3 \sqrt{\pi} \Gamma\left(\frac{3}{2}\right) \Gamma\left(\frac{7}{4}\right)}{\Gamma\left(\frac{9}{4}\right)}, \\
k_{5}= & \frac{-2}{\sqrt{3}} .
\end{aligned}
$$

The constants $c_{1}$ and $c_{2}$ appearing in the Eq. (30) are given as follows

$$
c_{1}=\frac{\sqrt{\pi}}{2} \frac{\Gamma\left(\frac{3}{4}\right)}{\Gamma\left(\frac{5}{4}\right)}+\sum_{n=1}^{\infty}\left(\frac{\Gamma\left(n+\frac{1}{2}\right)}{2 \Gamma(n+1)} \frac{\Gamma\left(\frac{3 n+3}{4}\right)}{\Gamma\left(\frac{3 n+5}{4}\right)}-\frac{1}{\sqrt{3} n}\right),
$$




$$
\begin{aligned}
c_{2}= & \frac{1}{\sqrt{3}}-\frac{3}{2} \sum_{n=0}^{\infty}\left(\frac{\Gamma\left(n+\frac{3}{2}\right)}{\Gamma(n+1)} \frac{\Gamma\left(\frac{3 n+6}{4}\right)}{\Gamma\left(\frac{3 n+8}{4}\right)}-\frac{2}{\sqrt{3}}\right) \\
& +\frac{3}{2} \sum_{n=0}^{\infty}\left(\frac{\Gamma\left(n+\frac{3}{2}\right)}{\Gamma(n+1)} \frac{\Gamma\left(\frac{3 n+7}{4}\right)}{\Gamma\left(\frac{3 n+9}{4}\right)}-\frac{2}{\sqrt{3}}\right) .
\end{aligned}
$$

A.2 Non-extremal RN-AdS 4 (large charge-high temperature)

The constants $K_{1}^{\prime}$ and $K_{2}^{\prime}$ in the Eq. (33) are given as follows

$$
\begin{aligned}
K_{1}^{\prime}= & -\frac{2 \sqrt{\pi} \Gamma\left(\frac{3}{4}\right)}{\Gamma\left(\frac{1}{4}\right)}+\frac{\log [4]-10}{8} \\
& +\frac{1}{2} \sum_{n=2}^{\infty}\left(\frac{1}{n-1} \frac{\Gamma\left(n+\frac{1}{2}\right)}{\Gamma(n+1)} \frac{\Gamma\left(\frac{n+3}{4}\right)}{\Gamma\left(\frac{n+5}{4}\right)}-\frac{2}{n^{2}}\right)+\frac{\pi^{2}}{6} \\
K_{2}^{\prime}= & \frac{\pi^{2}}{6}-\frac{3}{2} .
\end{aligned}
$$

\section{A.3 Extremal RN-AdS4 (large charge )}

The constants $K_{1}, K_{2}$ and $K_{3}$ in the Eq. (39) are given as follows

$$
\begin{aligned}
K_{1}= & \frac{2}{\sqrt{6}}\left[-2 \frac{\sqrt{\pi} \Gamma\left(\frac{3}{4}\right)}{\Gamma\left(\frac{1}{4}\right)}+\frac{\log [4]}{4}\right. \\
& -\frac{1+2 \sqrt{\pi}}{2}+\sqrt{\pi} \zeta\left(\frac{3}{2}\right) \\
+ & \left.\frac{\sqrt{\pi}}{2} \sum_{n=2}^{\infty}\left(\frac{1}{n-1} \frac{\Gamma\left(\frac{n+3}{4}\right)}{\Gamma\left(\frac{n+5}{4}\right)}-\frac{2}{n \sqrt{n}}\right)\right], \\
K_{2}= & -\frac{2 \pi}{\sqrt{6}}, \\
K_{3}= & \frac{2}{\sqrt{6}}\left[1-\sqrt{\pi}+\sqrt{\pi} \zeta\left(\frac{3}{2}\right)\right] .
\end{aligned}
$$

\section{Appendix B: Non-extremal and extremal RN-AdS $\mathrm{S}_{\mathrm{d}+1}$}

B.1 Non-extremal RN-AdS $\mathrm{d}+1_{\text {(Small chemical }}$ potential-low temperature)

The constants $\mathcal{S}_{0}$ and $\mathcal{S}_{1}$ appearing in the Eq. (64) are given as follows

$$
\begin{aligned}
& \mathcal{S}_{0}=\frac{2^{d-2} \pi^{\frac{d-1}{2}} \Gamma\left(-\frac{d-2}{2(d-1)}\right)}{(d-1) \Gamma\left(\frac{1}{2(d-1)}\right)}\left(\frac{\Gamma\left(\frac{d}{2(d-1)}\right)}{\Gamma\left(\frac{1}{2(d-1)}\right)}\right)^{d-2}, \\
& \mathcal{S}_{1}=\frac{\Gamma\left(\frac{1}{2(d-1)}\right)^{d+1} 2^{-d-1} \pi^{-\frac{d}{2}}}{\Gamma\left(\frac{d}{2(d-1)}\right)^{d} \Gamma\left(\frac{1}{2}+\frac{1}{d-1}\right)}
\end{aligned}
$$

$$
\left(\frac{\Gamma\left(\frac{1}{d-1}\right)}{\Gamma\left(-\frac{d-2}{2(d-1)}\right)}+\frac{2^{\frac{1}{d-1}}(d-2) \Gamma\left(1+\frac{1}{2(d-1)}\right)}{\sqrt{\pi}(d+1)}\right) .
$$

B.2 Non-extremal RN-AdS $\mathrm{d}+1_{\text {(small chemical }}$ potential-high temperature)

The function $\gamma_{d}\left(\frac{\mu}{T}\right)$ appearing in the Eq. (66) is given as follows

$$
\begin{aligned}
\gamma_{d}\left(\frac{\mu}{T}\right)= & N(1)+\frac{d^{2}(d-2)}{16 \pi^{2}(d-1)}\left(\frac{\mu}{T}\right)^{2} \int_{0}^{1} d x \\
& \times\left(\frac{x \sqrt{1-x^{2(d-1)}}}{\sqrt{1-x^{d}}}\right)\left(\frac{1-x^{d-2}}{1-x^{d}}\right)+\mathcal{O}\left(\frac{\mu}{T}\right)^{4},
\end{aligned}
$$

where the numerical constant $N(\varepsilon)$ is given as

$$
\begin{aligned}
N(\varepsilon)= & 2\left[\frac{\sqrt{\pi} \Gamma\left(-\frac{d-2}{2(d-1)}\right)}{2(d-1) \Gamma\left(\frac{1}{2(d-1)}\right)}\right]+2 \int_{0}^{1} d x \\
& \times\left(\frac{\sqrt{1-x^{2(d-1)}}}{x^{d-1} \sqrt{f\left(z_{H} x\right)}}-\frac{1}{x^{d-1} \sqrt{1-x^{2(d-1)}}}\right) .
\end{aligned}
$$

B.3 Non-extremal RN-AdS $\mathrm{S}_{\mathrm{d}+1}$ (large chemical potential-low temperature)

The numerical constants $N_{0}, N_{1}$ in the Eq. (68) are given as follows

$$
\begin{aligned}
N_{0}= & 2\left[\frac{\sqrt{\pi} \Gamma\left(-\frac{d-2}{2(d-1)}\right)}{2(d-1) \Gamma\left(\frac{1}{2(d-1)}\right)}\right]+2 \int_{0}^{1} d x \\
& \left(\frac{\sqrt{1-x^{2(d-1)}}}{x^{d-1} \sqrt{1-b_{0} x^{d}+b_{1} x^{2(d-1)}}}\right) \\
& \left.-\frac{1}{x^{d-1} \sqrt{1-x^{2(d-1)}}}\right), \\
N_{1}= & \left.\int_{0}^{1} \frac{d x\left(\frac{x \sqrt{1-x^{2(d-1)}}}{\sqrt{1-b_{0} x^{d}+b_{1} x^{2(d-1)}}}\right)}{1-x^{d-2}}\right) .
\end{aligned}
$$

\section{References}

1. P. Calabrese, J.L. Cardy, Entanglement entropy and quantum field theory. J. Stat. Mech. 0406, P06002 (2004). arXiv:hep-th/0405152 [hep-th]

2. P. Calabrese, J. Cardy, Entanglement entropy and conformal field theory. J. Phys. A 42, 504005 (2009). arXiv:0905.4013 [condmat.stat-mech] 
3. S. Ryu, T. Takayanagi, Holographic derivation of entanglement entropy from AdS/CFT. Phys. Rev. Lett. 96, 181602 (2006). arXiv:hep-th/0603001 [hep-th]

4. S. Ryu, T. Takayanagi, Aspects of holographic entanglement entropy. JHEP 08, 045 (2006). arXiv:hep-th/0605073 [hep-th]

5. T. Nishioka, S. Ryu, T. Takayanagi, Holographic entanglement entropy: An overview. J. Phys. A 42, 504008 (2009). arXiv:0905.0932 [hep-th]

6. T. Takayanagi, Entanglement entropy from a holographic viewpoint. Class. Quant. Grav. 29, 153001 (2012). arXiv:1204.2450 [gr-qc]

7. M. Cadoni, M. Melis, "Entanglement entropy of ads black holes," Entropy 12(11), 2244-2267 (2010). http://www.mdpi.com/ $1099-4300 / 12 / 11 / 2244$

8. D.D. Blanco, H. Casini, L.-Y. Hung, R.C. Myers, Relative entropy and holography. JHEP 08, 060 (2013). arXiv:1305.3182 [hep-th]

9. W. Fischler, S. Kundu, Strongly coupled gauge theories: High and low temperature behavior of non-local observables. J. High Energy Phys. 2013(5), 98 (2013). https://doi.org/10.1007/ JHEP05(2013)098

10. W. Fischler, A. Kundu, S. Kundu, Holographic mutual information at finite temperature. Phys. Rev. D 87(12), 126012 (2013). arXiv:1212.4764 [hep-th]

11. P. Chaturvedi, V. Malvimat, G. Sengupta, Entanglement thermodynamics for charged black holes. Phys. Rev. D 94(6), 066004 (2016). arXiv:1601.00303 [hep-th]

12. D.V. Fursaev, Proof of the holographic formula for entanglement entropy. JHEP 09, 018 (2006). arXiv:hep-th/0606184 [hep-th]

13. M. Headrick, Entanglement renyi entropies in holographic theories. Phys. Rev. D 82, 126010 (2010). arXiv:1006.0047 [hep-th]

14. T. Hartman, "Entanglement entropy at large central charge," arXiv:1303.6955 [hep-th]

15. T. Faulkner, "The Entanglement Renyi Entropies of Disjoint Intervals in AdS/CFT," arXiv:1303.7221 [hep-th]

16. H. Casini, M. Huerta, R.C. Myers, Towards a derivation of holographic entanglement entropy. JHEP 05, 036 (2011). arXiv: 1102.0440 [hep-th]

17. A. Lewkowycz, J. Maldacena, Generalized gravitational entropy. JHEP 08, 090 (2013). arXiv:1304.4926 [hep-th]

18. G. Vidal, R.F. Werner, Computable measure of entanglement. Phys. Rev. A 65, 032314 (2002). https://doi.org/10.1103/PhysRevA.65. 032314

19. M.B. Plenio, Logarithmic negativity: A full entanglement monotone that is not convex. Phys. Rev. Lett. 95(9), 090503 (2005). arXiv:quant-ph/0505071 [quant-ph]

20. P. Calabrese, J. Cardy, E. Tonni, Entanglement negativity in extended systems: A field theoretical approach. J. Stat. Mech. 1302, P02008 (2013). arXiv:1210.5359 [cond-mat.stat-mech]

21. P. Calabrese, J. Cardy, E. Tonni, Entanglement negativity in quantum field theory. Phys. Rev. Lett. 109, 130502 (2012). arXiv:1206.3092 [cond-mat.stat-mech]

22. P. Calabrese, J. Cardy, E. Tonni, Finite temperature entanglement negativity in conformal field theory. J. Phys. A 48(1), 015006 (2015). arXiv:1408.3043 [cond-mat.stat-mech]

23. M. Rangamani, M. Rota, Comments on entanglement negativity in holographic field theories. JHEP 10, 060 (2014). arXiv:1406.6989 [hep-th]
24. E. Perlmutter, M. Rangamani, M. Rota, Central charges and the sign of entanglement in 4D conformal field theories. Phys. Rev. Lett. 115(17), 171601 (2015). arXiv:1506.01679 [hep-th]

25. P. Chaturvedi, V. Malvimat, G. Sengupta, "Holographic quantum entanglement negativity," arXiv:1609.06609 [hep-th]

26. P. Chaturvedi, V. Malvimat, G. Sengupta, "Entanglement negativity, holography and black holes," arXiv:1602.01147 [hep-th]

27. P. Chaturvedi, V. Malvimat, G. Sengupta, "Covariant holographic entanglement negativity," arXiv:1611.00593 [hep-th]

28. V. Malvimat, G. Sengupta, "Entanglement negativity at large central charge," arXiv:1712.02288 [hep-th]

29. P. Jain, V. Malvimat, S. Mondal, G. Sengupta, "Holographic entanglement negativity conjecture for adjacent intervals in $A d S_{3} / C F T_{2}$, , arXiv:1707.08293 [hep-th]

30. A. Coser, E. Tonni, P. Calabrese, Entanglement negativity after a global quantum quench. J. Stat. Mech. 1412(12), P12017 (2014). arXiv:1410.0900 [cond-mat.stat-mech]

31. X. Wen, P.-Y. Chang, S. Ryu, Entanglement negativity after a local quantum quench in conformal field theories. Phys. Rev. B 92(7), 075109 (2015). arXiv: 1501.00568 [cond-mat.stat-mech]

32. P. Jain, V. Malvimat, S. Mondal, and G. Sengupta, "Holographic entanglement negativity for adjacent subsystems in $\mathrm{AdS}_{\mathrm{d}+1} / \mathrm{CFT}_{\mathrm{d}}$, , arXiv:1708.00612 [hep-th]

33. W. Fischler, S. Kundu, Strongly coupled gauge theories: High and low temperature behavior of non-local observables. JHEP 05, 098 (2013). arXiv:1212.2643 [hep-th]

34. C. De Nobili, A. Coser, E. Tonni, Entanglement negativity in a two dimensional harmonic lattice: Area law and corner contributions. J. Stat. Mech. 1608(8), 083102 (2016). arXiv:1604.02609 [condmat.stat-mech]

35. N.E. Sherman, T. Devakul, M.B. Hastings, R.R.P. Singh, Nonzerotemperature entanglement negativity of quantum spin models: Area law, linked cluster expansions, and sudden death. Phys. Rev. E 93, 022128 (2016). 10.1103/PhysRevE.93.022128

36. P. Jain, V. Malvimat, S. Mondal, G. Sengupta, "Covariant holographic entanglement negativity conjecture for adjacent subsystems in $\mathrm{AdS}_{\mathrm{d}+1} / \mathrm{CFT}_{\mathrm{d}}$," arXiv:1710.06138 [hep-th]

37. S. Kundu, J.F. Pedraza, Aspects of holographic entanglement at finite temperature and chemical potential. JHEP 08, 177 (2016). arXiv: 1602.07353 [hep-th]

38. J.D. Brown, M. Henneaux, Central charges in the canonical realization of asymptotic symmetries: An example from threedimensional gravity. Comm. Math. Phys. 104(2), 207-226 (1986) https://projecteuclid.org:443/euclid.cmp/1104114999

39. V. Malvimat, S. Mondal, B. Paul, G. Sengupta, "Holographic entanglement negativity for disjoint intervals in $A d S_{3} / C F T_{2}$," arXiv: 1810.08015 [hep-th] 